\title{
Efecto de la bupivacaína versus bupivacaína más dexmedetomidina intratecal sobre el dolor posquirúrgico
}

\author{
Effect of bupivacaine versus bupivacaine plus intrathecal \\ dexmedetomidine in postoperative pain
}

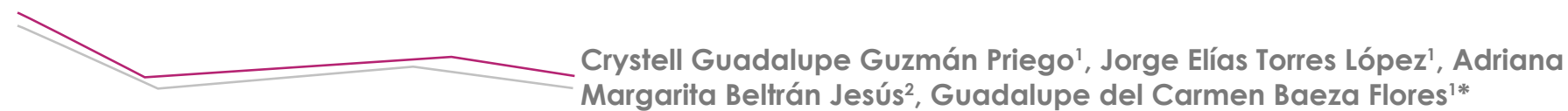

Guzmán Priego, C. G., Torres López, J. E., Beltrán Jesús, A. M., Baeza Flores, G. C. Efecto de la bupivacaína versus bupivacaína más dexmedetomidina intratecal sobre el dolor posquirúrgico. Investigación y Ciencia de la Universidad Autónoma de Aguascalientes. Número 70: 38-43, enero-abril 2017.

\section{RESUMEN}

La anestesia regional ha demostrado grandes beneficios asociados al uso combinado de dexmedetomidina y bupivacaína, por lo que cuantificar el efecto analgésico de bupivacaína vs. bupivacaína más dexmedetomidina intratecal en el control del dolor posquirúrgico se realizó a través de un ensayo clínico controlado. A muestra de 60 sujetos divididos aleatoriamente; Grupo b: recibieron bupivacaína a $0.5 \%$ en una dosis de $0.2 \mathrm{mg} / \mathrm{kg}$ vía intratecal con $0.5 \mathrm{ml}$ de solución fisiológica y Grupo d: recibieron bupivacaína a $0.5 \%$ en una dosis de $0.2 \mathrm{mg} /$ $\mathrm{kg}$ vía intratecal con $5 \mathrm{\mu g}$ de dexmedetomidina en $0.5 \mathrm{ml}$ de solución fisiológica. Derivado de la aleatorización y examen de los sujetos se obtuvo que el inicio del bloqueo apareció en el grupo con dexmedetomidina $(8.5 \pm 1.0 \mathrm{~min})$ con respecto a bupivacaína (9.9 $\pm 1.8 \mathrm{~min})(p=0.0006)$ y se observó mayor duración de analgesia posoperatoria con un valor $p$ de 0.0230 , lo que lleva a concluir que el uso de dexmedetomidina proporciona beneficios en el control del dolor posquirúrgico.

Palabras clave: dolor posoperatorio, anestesia, anestésicos, bupivacaína, dexmedetomidina.

Keywords: postoperative pain, anesthesia, anesthetics, bupivacaine, dexmedetomidine.

Recibido: 18 de junio de 2016, aceptado: 16 de diciembre de 2016

Universidad Juárez Autónoma de Tabasco, México.

Hospital Regional de Alta Especialidad Juan Graham Casasús, México.

Autor para correspondencia: ba lupitabaeza@hotmail.com
ABSTRACT

Regional anesthesia has shown great benefits associated with the combined use of dexmedetomidine and bupivacaine. Therefore, quantifying the analgesic effect of bupivacaine versus bupivacaine plus intrathecal dexmedetomidine in the control of postoperative pain was performed through a controlled clinical trial. In a sample of 60 randomly divided subjects; Group b: received $0.5 \%$ bupivacaine at a dose of $0.2 \mathrm{mg} / \mathrm{kg}$ intrathecally with $0.5 \mathrm{ml}$ of physiological solution and Group d: received 0.5\% bupivacaine at a dose of $0.2 \mathrm{mg} / \mathrm{kg}$ via intrathecal administration with $5 \mu \mathrm{g}$ of dexmedetomidine in 0.5 $\mathrm{ml}$ of saline solution. From the randomization and evaluation of subjects, it was found that the onset of blockade ocurred in the group with dexmedetomidine (8.5 \pm 1.0 minutes) compared to bupivacaine $(9.9 \pm 1.8$ $\min )(p=0.0006)$ and a longer duration of postoperative analgesia with a $p$-value of 0.0230 , that leads us to conclude that the use of dexmedetomidine provides benefits in the control of postoperative pain.

\section{INTRODUCCIÓN}

El dolor es definido como "una experiencia sensorial y emocional desagradable, asociada con daño tisular real o potencial que puede ser descrita en términos de dicho daño" (Anand y Craig, 1996). De acuerdo con las distintas clasificaciones de dolor, es objeto de nuestra atención el dolor posoperatorio, que aparece como consecuencia 


\section{InVESTIGACIÓn Y CIERCIA DE LA UחIVERSIDAD AUTÓnOMA DE AGUASCALIENTES}

de la estimulación nociceptiva resultante de la intervención quirúrgica sobre los distintos órganos y tejidos, cuya duración es limitada y su intensidad máxima se presenta en las primeras $24 \mathrm{~h}$ con una disminución progresiva (Eberhard y Mora, 2004; Reyes Chiquete, 2005). La agresión quirúrgica, además de activar las vías nociceptivas, origina una respuesta neurohumoral generalizada, mediada por el eje hipotálamo-hipófisis suprarrenal, una reacción de defensa del organismo frente a la lesión tisular. El sistema inmune y los cambios hormonales (liberación de corticoesterona, progesterona, catecolaminas, etc.) transmiten información entre la periferia y el sistema nervioso, esta comunicación bidireccional tiene como función proteger la integridad del organismo (Wrona, 2006).

La ausencia temporal de sensibilidad para una intervención quirúrgica es provista por sedación, anestesia general, regional o local, dependiendo del tipo de procedimiento que se realice (Salamanca et al., 2009). En el caso de anestesia regional espinal se ha observado efectividad, rapidez, seguridad y mejor manejo del dolor (Rebollo Manrique, 2013) asociado al uso de anestésicos locales (Valencia Gómez y García Araque, 2011) como bupivacaína (Santana Hernández et al., 2010).

Estudios anteriores demuestran que la dexmedetomidina aplicada por vía intravenosa antes de la anestesia disminuyó la intensidad del dolor y consumo de analgésicos en periodo posoperatorio (Del Angel García et al., 2006), que su adición a ropivacaína administrada vía intratecal prolonga la duración del bloqueo motor y sensorial (Gupta et al., 2011) y tambien hay evidencia de inicio más rápido de anestesia, sin presencia de efectos adversos (Shukla et al., 2011). Derivado de lo anterior, el objetivo del presente estudio fue cuantificar el efecto analgésico de bupivacaína vs. bupivacaína más dexmedetomidina intratecal en el control del dolor posquirúrgico.

\section{MATERIALES Y MÉTODOS}

Con registro en el Acta no. 275 se obtuvo la autorización del Comité de Investigación institucional de la Universidad Juárez Autónoma de Tabasco y del Hospital Regional de Alta Especialidad Dr. Juan Graham Casasús. Se realizó un diseño experimental de tipo ensayo clínico controlado, en pacientes programados para cirugía de abdomen inferior o extremidades inferiores.

Se estudió una muestra de 60 sujetos programados a cirugía durante el periodo comprendido desde el 1 de octubre hasta el 15 de diciembre de 2015, que aceptaran participar en el estudio a través de firma de consentimiento bajo información. Los sujetos que participaron en el estudio se dividieron en dos grupos asignados aleatoriamente; Grupo b: recibieron bupivacaína a $0.5 \%$ en una dosis de $0.2 \mathrm{mg} / \mathrm{kg}$ vía intratecal con $0.5 \mathrm{ml}$ de solución fisiológica y Grupo d: recibieron bupivacaína a $0.5 \%$ en una dosis de $0.2 \mathrm{mg} / \mathrm{kg}$ vía intratecal con $5 \mu \mathrm{g}$ de dexmedetomidina en $0.5 \mathrm{ml}$ de solución fisiológica.

Después de la cirugía permanecieron en el área de recuperación, para evitar sesgos; en la recolección de datos se designó personal capacitado ajeno a la investigación con instrucciones de registrar

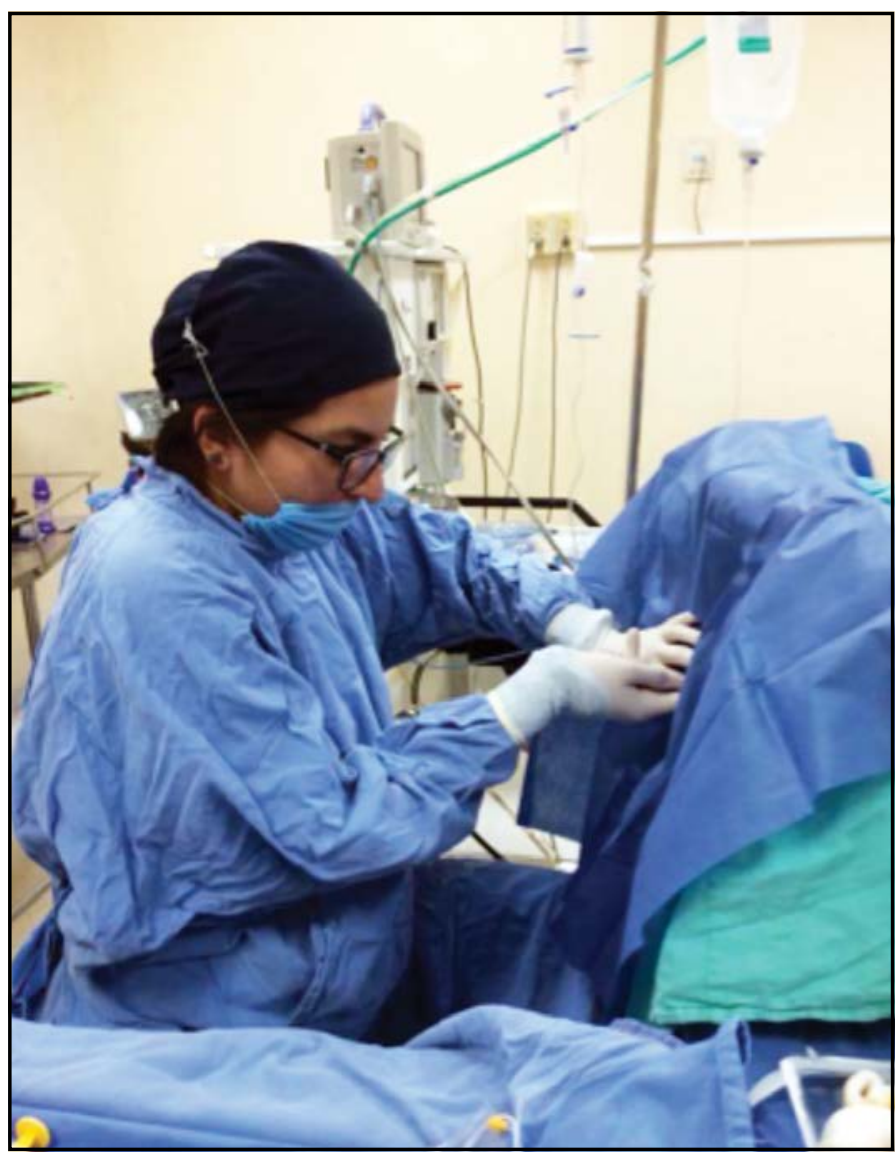

Figura 1. Bloqueo intratecal. Paciente en decúbito lateral izquierdo. Médico especialista en anestesiología previa asepsia, antisepsia y colocación de campos estériles lleva a cabo la punción lumbar a nivel de espacio L1-L2. Fotografía tomada por María José Ayala Alcocer. 
IIVESTIGACIÓn Y CIERCIA DE LA UNIVERSIDAD AUTÓNOMA DE RGUASCALIERTES signos vitales cada hora durante las primeras 4 h y posteriormente cada 4 hasta su egreso del área. También se evaluó la presencia de dolor posoperatorio por medio de la escala visual análoga, frecuencia cardiaca, presión arterial sistólica y diastólica al momento de su ingreso a la sala de recuperación y a los 15, 30, 45, 60, 120, 240, 480, 720, 1080, 1440 min; por último, se registró si el personal de enfermería utilizó fármacos de analgesia de rescate en el posoperatorio para manejo del dolor en el sitio de la operación.

La información se registró en una base de datos en Microsoft Excel 2010 y se analizó por medio del programa Graphpad Prim 6 versión 6.07, a través de estadística descriptiva como medidas de tendencia central y dispersión. El análisis de intensidad de dolor posoperatorio se efectuó a través de la escala visual análoga (EVA) por medio de estadística no paramétrica mediante la prueba estadística de Mann-Whitney. Para las posibles diferencias entre variables cuantitativas se utilizó la prueba $\dagger$ de Student no pareada; finalmente, para evaluar la presencia y ausencia de dolor se realizó la prueba chi-cuadrada con una $p<0.05$ como estadísticamente significativa.

\section{RESULTADOS}

Se incluyeron 60 sujetos que fueron sometidos a cirugía de abdomen inferior y extremidades inferiores, 30 por cada grupo. Del Grupo b se obtuvo una media edad de $48 \pm 12.49$ años, $53.3 \%(n=16)$ de sexo femenino, con una media de $78.17 \pm 13.64$ $\mathrm{kg}$ de peso, $1.64 \pm 0.074 \mathrm{~m}, 28.93 \pm 4.06$ de IMC y en lo que corresponde a las características del Grupo d se obtuvo una media de edad de $51.30 \pm 12.32$ años, $53.3 \%(n=16)$ de sexo femenino, $73.70 \pm 11.43$ $\mathrm{kg}$ de peso, $1.61 \pm 0.077 \mathrm{~m}, 28.32 \pm 3.39$ de IMC, tal como se observa en la Tabla 1.

\section{Parámetros anestésicos}

En la Tabla 2 se observa que la duración de la cirugía para el grupo bupivacaína fue de $64.4 \pm 23.3$ min y de $69.3 \pm 17.4$ min para el grupo dexmedetomidina. Por otro lado, el tiempo de inicio del bloqueo apareció en el grupo con dexmedetomidina a los $8.5 \pm 1.0 \mathrm{~min}$, el tiempo de regresión del bloqueo en el grupo con dexmedetomidina fue de $92.0 \pm 15.82$ min y el tiempo de terminación del bloqueo en el grupo tratado con dexmedetomidina $161.8 \pm 14.20$, comparado con $100.6 \pm 27.44$ min del grupo con bupivacaína.

En la evaluación del dolor posoperatorio por medio de la Escala Visual Análoga, los pacientes tratados con dexmedetomidina refirieron dolor hasta los 30 min del posoperatorio (Figura 2), mientras que en los tratados con bupivacaína el dolor se presentó inmediatamente en el posoperatorio; al realizar la prueba $t$ de Student se encontró diferencia significativa $(p=0.0230)$, misma al comparar las áreas bajo la curva $(A B C)$ entre los grupos de tratamiento $(p<0.0260)$.

El rescate analgésico después de la operación (Tabla 3) se realizó a 26 pacientes en el grupo con bupivacaína, mientras que en el grupo con dexmedetomidina solamente en 14, lo que resultó estadísticamente significativo ( $p<0.0022)$, de la misma forma se observó que el tiempo de la analgesia de rescate se prolongó en más de 120 min en el caso de la dexmedetomidina cuando se comparó con la bupivacaína ( $p<0.0001$ ); además,

Tabla 1. Datos demográficos

\begin{tabular}{|l|c|c|c|}
\hline Características & $\begin{array}{c}\text { Grupo bupivacaína } \\
(\boldsymbol{n}=\mathbf{3 0})\end{array}$ & $\begin{array}{c}\text { Grupo dexmedetomidina } \\
(\boldsymbol{n}=\mathbf{3 0})\end{array}$ & Valor de $\boldsymbol{p}$ \\
\hline Edad (años) & $48 \pm 12.49$ & $51.30 \pm 12.32$ & 0.307 \\
\hline Género (F:M) & $16: 14$ & $16: 14$ & 1 \\
\hline Peso (kg) & $78.17 \pm 13.64$ & $73.70 \pm 11.43$ & 0.1744 \\
\hline Talla (m) & $1.64 \pm 0.074$ & $1.61 \pm 0.077$ & 0.14 \\
\hline IMC & $28.93 \pm 4.06$ & $28.32 \pm 3.39$ & 0.5286 \\
\hline
\end{tabular}

Las variables cuantitativas se expresan como media y desviación estándar. Las variables categóricas se presentan como $n$. Para la comparación entre grupos se realizó la prueba t de Student no pareada para variables cuantitativas y la chi-cuadrada para variables cualitativas. Se consideraron estadísticamente significativos valores de $p<0.05$. Elaboración propia. 
InVESTIGACIÓn Y GIEnCIR DE

LA UNIVERSIDAD AUTÓOOOMA

DE RGUASCALIETTES

Tabla 2. Parámetros intraoperatorios y anestésicos

\begin{tabular}{|l|c|c|c|}
\hline Características & $\begin{array}{c}\text { Grupo bupivacaína } \\
(\boldsymbol{n}=\mathbf{3 0 )}\end{array}$ & $\begin{array}{c}\text { Grupo } \\
\text { dexmedetomidina } \\
(\boldsymbol{n}=\mathbf{3 0})\end{array}$ & Valor de $\mathbf{p}$ \\
\hline Duración cirugía (min) & $64.4 \pm 23.3$ & $69.3 \pm 17.4$ & 0.1375 \\
\hline Inicio de bloqueo (min) & $9.9 \pm 1.81$ & $8.5 \pm 1.01$ & 0.0006 \\
\hline Regresión de bloqueo (min) & $59.1 \pm 15.45$ & $92.0 \pm 15.82$ & $<0.0001$ \\
\hline Término de bloqueo (min) & $100.6 \pm 27.44$ & $161.8 \pm 14.20$ & $<0.0001$ \\
\hline
\end{tabular}

Los datos se presentan como media y desviación estándar. Se realizó una prueba t de Student no pareada, si se consideran estadísticamente significativos aquellos valores de $p<0.05$. Elaboración propia.

Tabla 3. Rescate analgésico posoperatorio

\begin{tabular}{|l|c|c|c|}
\hline Características & $\begin{array}{c}\text { Grupo bupivacaína } \\
(\boldsymbol{n}=\mathbf{3 0})\end{array}$ & $\begin{array}{c}\text { Grupo dexmedetomidina } \\
(\boldsymbol{n}=\mathbf{3 0})\end{array}$ & Valor de $\boldsymbol{p}$ \\
\hline Pacientes rescatados (\%) & $26(86.7)$ & $14(46.7)$ & 0.0022 \\
\hline Tiempo rescate & $15.58 \pm 12.36$ & $197.1 \pm 59.67$ & $<0.0001$ \\
\hline $\begin{array}{l}\text { Intensidad de dolor (EVA) al } \\
\text { momento del rescate }\end{array}$ & $5.7 \pm 1.59$ & $3.7 \pm 1.27$ & 0.0008 \\
\hline
\end{tabular}

Los datos se presentan como media y desviación estándar o como $n$ y (porcentaje). Para las variables cualitativas se utilizó la prueba chi-cuadrada $\left(X^{2}\right)$. Para las variables cuantitativas se realizó una prueba $\dagger$ de Student no pareada, considerando estadísticamente significativos aquellos valores de $p<0.05$. Elaboración propia.

los puntajes de la EVA al momento de la analgesia de rescate para el grupo con dexmedetomidina fueron de $3.71 \pm 1.27$ y en el grupo con bupivacaína de $5.7 \pm 1.59$, la diferencia de dos puntos de la EVA $(p=0.0008)$ resultó significativa, lo que demuestra que la dexmedetomidina es efectiva para prolongar la analgesia posoperatoria y disminuir los requerimientos de analgesia.

La frecuencia de efectos adversos en ambos grupos fue similar para los dos grupos, como se muestra en la Tabla 4.

Tabla 4. Efectos adversos

\begin{tabular}{|l|c|c|c|}
\hline $\begin{array}{l}\text { Efecto } \\
\text { adverso }\end{array}$ & Bupivacaína & Dexmedetomidina & $\boldsymbol{p}$ \\
\hline Náuseas & $0(0)$ & $3(10)$ & 0.2373 \\
\hline Vómito & $0(0)$ & $0(0)$ & 1.000 \\
\hline Bradicardia & $2(6.7)$ & $1(3.3)$ & 1.000 \\
\hline Hipotensión & $4(13.3)$ & $6(20)$ & 0.7306 \\
\hline
\end{tabular}

Los datos se presentan como frecuencia y (porcentaje). Se realizó una prueba chi-cuadrada $\left(X^{2}\right)$ o prueba exacta de Fisher para la comparación entre grupos. Se consideraron estadísticamente significativos valores $p<0.05$. Elaboración propia.

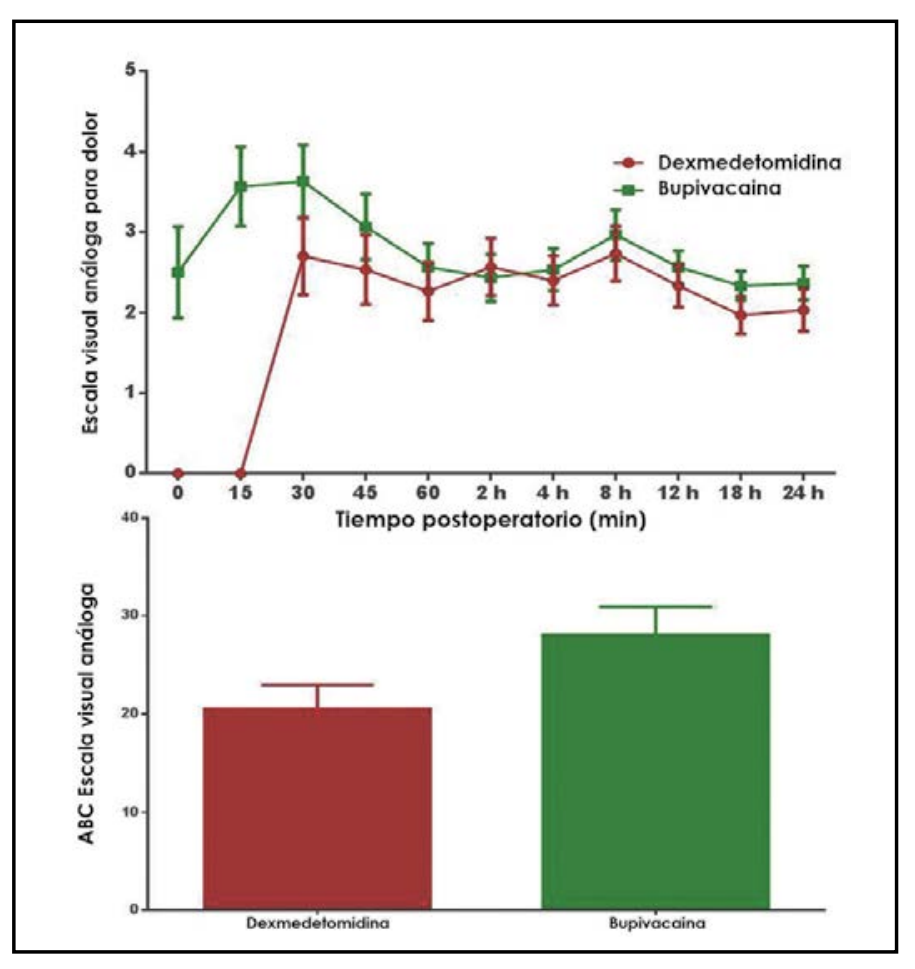

Figura 2. Valoración del dolor posoperatorio de la EVA. Los datos se presentan como media y error estándar para los grupos. Elaboración propia. 
IIVESTIGACIÓn Y CIERCIA DE LA UNIVERSIDAD AUTÓNOMA DE RGUASCALIERTES

En esta investigación se comparó el efecto de la dexmedetomidina asociada a la bupivacaína con la administración de bupivacaína sola por vía intratecal en pacientes sometidos a cirugía de abdomen inferior y extremidades inferiores, por lo que a través de nuestros resultados se sugiere que la dexmedetomidina es efectiva para prolongar la analgesia posoperatoria y disminuir los requerimientos de analgesia; sin embargo, para mantener la calidad del mismo, la limitación principal fue la disponibilidad de la dexmedetomidina a nivel institucional debido a su costo.

Se encontró que el grupo tratado con dexmedetomidina presentó un inicio más rápido del bloqueo motor y sensorial, mientras que los tiempos de regresión y término del bloqueo se prolongaron de forma significativa cuando se compararon con el grupo con bupivacaína, esto concuerda con lo reportado por Gupta et al. (2011), ya que ellos encontraron resultados similares a los de este estudio.

En el caso del efecto de la dexmedetomidina sobre la analgesia posterior a la operación, varios autores han reportado disminuciones en el puntaje de la escala análoga visual, menores requerimientos en las dosis de analgesia de rescate y una prolongación en el tiempo de administración necesaria de la misma en los pacientes a los que se ha medicado con dexmedetomidina (Fernández Galinski et al., 1996; Del Angel García et al., 2006; Kanazi et al., 2006; Vaca Damy et al., 2011); en este trabajo se presentó un incremento de casi $2 \mathrm{~h}$ en el tiempo de administración de analgesia de rescate y un menor número de pacientes que requirieron rescate analgésico.

Los efectos adversos presentados por los pacientes tratados con dexmedetomidina fueron las náuseas, bradicardia e hipotensión; sin embargo, la frecuencia de estos efectos adversos no tuvo diferencia significativa al compararla con la de los anestesiados con bupivacaína; lo anterior resulta similar a lo reportado por los demás trabajos, lo que demuestra que la administración de la dexmedetomidina en dosis de $5 \mu \mathrm{g}$ por vía intratecal es segura y efectiva para el manejo del dolor estudiado (Fernández Galinski et al., 1996; Gupta et al., 2011; Shukla et al., 2011; Vaca Damy et al., 2011).

Aun con evidencia del papel que tiene la adición de dexmedetomidina a bupivacaína en el control del dolor posterior a una cirugía se recomienda la realización de más estudios que valoren la eficacia, seguridad y costos para tener un panorama sobre los beneficios y repercusiones que tendrá sobre la dinámica institucional y la posibilidad de aplicar la recomendación.

CONCLUSIONES

La dexmedetomidina a dosis de $5 \mu \mathrm{g}$ asociada a la bupivacaína administrada por vía intratecal es más efectiva en la analgesia posoperatoria comparada con esta sustancia sola en cirugía de abdomen inferior y extremidades inferiores, presenta un tiempo más prolongado de analgesia posoperatoria, con requerimiento de analgesia de rescate hasta $2 \mathrm{~h}$ después que en el grupo con bupivacaína.

Esta investigación se realizó bajo apego estricto a la Ley General de Salud en México, categorizado tipo II, con riesgo mínimo. 
InVESTIGAGIÓn Y CIERCIA DE

LA UNIVERSIDAD AUTÓNOTHR

DE RGUASCALIETTES

LITERATURA CITADA

\section{De páginas electrónicas}

- ANAND, K. J. S. y CRAIG, K. D. New perspectives on the definition of pain. Pain, 67(1): 3-6, 1996. doi: 10.1016/03043959(96)03135-1

- Del ANGel GARCíA, R. et al. Analgesia preventiva postoperatoria con dexmedetomidina en hernioplastia inguinal. Gaceta Médica de México, 142(1): 9-12, 2006. Recuperado de http://www.scielo.org.mx/scielo. php?script=sci_arttext\&pid=\$0016-38132006000100002

- EBerhard, M. E. y MORA, X. Manejo del dolor en el paciente pediátrico. Revista Chilena de Pediatría, 75(3): 277-279, 2004. Recuperado de http://www.scielo.cl/scielo.php?script=sci_ar ttext\&pid=S0370-41062004000300012

- FERNÁNDEZ GALINSKI, D. et al. Spinal anesthesia with bupivacaine and fentanyl in geriatric patients. Anesthesia \& Analgesia, 83(3): 537-541, 1996. Recuperado de http://journals. Iww.com/anesthesia-analgesia/Abstract/1996/09000/Spinal_ Anesthesia_with_Bupivacaine_and_Fentanyl_in.17.aspx

- GUPTA, R. et al. Dexmedetomidine as an intrathecal adjuvant for postoperative analgesia. Indian Journal of Anaesthesia, 55(4): 347-351, 2011 . doi: 10.4103/0019-5049.84841

- KANAZI, G. E. et al. Effect of low-dose dexmedetomidine or clonidine on the characteristics of bupivacaine spinal block. Acta Anaesthesiologica Scandinavica, 50(2): 222-227, 2006. doi: $10.1111 /$ j.1399-6576.2006.00919.x

- Rebollo MANRIQUe, R. E. Bloqueo subaracnoideo: una técnica para siempre. Revista Mexicana de Anestesiología, 36(Suppl. 1): S145-S149, 2013. Recuperado de http://www. medigraphic.com/pdfs/rma/cma-2013/cmas131af.pdf

- REYES CHIQUETE, D. Dolor agudo en el paciente con cáncer. Revista Mexicana de Anestesiología, 28(1): 175-176, 2005.
Recuperado de http://new.medigraphic.com/cgi-bin/ resumen.cgi? IDARTICULO=8269

- salamancA, R. N. et al. Anestesia espinal para colecistectomia. Revista Colombiana de Anestesiología, 37(3): 264-271, 2009. doi: 10.1016/S0120-3347(09)73009-6

- SANTANA HERNÁNDEZ, D. A. et al. Eficacia de la analgesia epidural posoperatoria con fentanil y bupivacaína en comparación con buprenorfina y bupivacaína en pacientes sometidos a artroplastia de cadera. Revista de Especialidades Médico-Quirúrgicas, 15(4): 204-210, 2010. Recuperado de http://www.redalyc.org/pdf/473/47316086004.pdf

- SHUKLA, D. et al. Comparative study of intrathecal dexmedetomidine with intrathecal magnesium sulfate used as adjuvants to bupivacaine. Journal of Anaesthesiology Clinical Pharmacology, 27(4): 495-499, 2011 . doi: 10.4103/09709185.86594

- VACA DAMY, A. et al. Dexmedetomidina más bupivacaína frente a bupivacaína sola en infusión peridural para el control del dolor postquirúrgico. Revista Mexicana de Neurociencia, 12(4): 181-186, 2011 . Recuperado de http://new.medigraphic. com/cgi-bin/new/resumen.cgięIDARTICULO=4481 1 \&IDP UBLICACION=4631 \&IDREVISTA=82\&NOMBRE=Revista\%20 Mexicana\%20de\%20Neurociencia

- VAlEnCIA GÓMEZ, R. E. y GARCÍA ARAQUe, H. F. Toxicidad por anestésicos locales: revisión de la literatura. Revista Colombiana de Anestesiología, 39(1): 40-54, 2011. doi: 10.5554/rca.v39i1.160

- WRONA, D. Neural-immune interactions: an integrative view of the bidirectional relationship between the brain and immune systems. Journal of Neuroimmunology, 172(1-2): 38-58, 2006. doi: http://dx.doi.org/10.1016/j.jneuroim.2005.10.017 